\title{
Estimativa do custo do tratamento de câncer de pele tipo melanoma no Estado de São Paulo - Brasil *
}

\author{
An estimate of the cost of treating melanoma disease in the \\ state of Sao Paulo - Brazil
}

\author{
Reynaldo José Sant'Anna Pereira de Souza ${ }^{1}$ \\ Marcelo Lacerda Rezende ${ }^{3}$ \\ Etiene Marques Duarte ${ }^{5}$
}

\author{
Adriana Prest Mattedi ${ }^{2}$ \\ Marcelo de Paula Corrêa ${ }^{4}$
}

Resumo: FundaMENTOS: O impacto econômico do diagnóstico e do tratamento do melanoma cutâneo não tem sido analisado no Brasil. Uma vez que crescem tanto a incidência do câncer de pele quanto a preocupação com ele, é importante que isto seja avaliado.

OвJETIVO: O objetivo deste trabalho foi estimar o custo direto de diagnosticar e tratar em seus diversos estádios o melanoma cutâneo em número de casos ocorridos no Estado de São Paulo, entre 2000 e 2007. MÉToDos: Como modelo de procedimento, foi utilizado o projeto diretriz (Clinical Practice Guidelines in Oncology), editado pelo National Comprehensive Cancer Network (NCCN) e adequado aos procedimentos da Fundação SobecCan - Hospital do Câncer de Ribeirão Preto - SP. Os custos estimados se baseiam nos valores do tratamento médico pagos pelo setor público (Sistema Único de Saúde - SUS) e pelo setor privado (convênios) em 2007. Houve 2.740 casos diagnosticados e estadiados no Estado de São Paulo no período estudado.

REsuttados: O custo total de tratamento dos melanomas malignos diagnosticados no estádio inicial, em valores de 2007, foi estimado em $\mathrm{R} \$ 33.012 .725,10$ para o SUS e $\mathrm{R} \$ 76.133 .662,80$ para os convênios. Os estádios iniciais 0, I e II da doença compreendem aproximadamente 4,2\% (SUS) e 1, 3\% (convênios) do custo total; os estádios III e IV consomem $95,8 \%$ e $98,7 \%$ do custo total, respectivamente.

ConcLusã̃: Os resultados apresentados reforçam o argumento de que o diagnóstico do melanoma cutâneo em seus estádios iniciais reduz os custos de tratamento, gerando considerável economia tanto para o sistema público de saúde (SUS) quanto para o sistema privado (convênios).

Palavras-chave: Melanoma; Melanoma/economia; Melanoma/terapia; Neoplasias cutâneas; Neoplasia cutâneas/economia; Raios ultravioleta

\begin{abstract}
OвJестіve: The purpose of this study was to estimate the direct cost of diagnosing and treating melanoma disease in Sao Paulo (Brazil) between the years 2000 and 2007.

Methods: The project Clinical Practice Guidelines in Oncology was used, adapted to the proceedings of SOBECCan Foundation. The estimated costs were based on the values of the medical treatment paid by the Brazilian National Health System (SUS) and private healthcare insurance companies (PHIC) in 2007. Results: The total cost was estimated in $\mathrm{R} \$ 33,012,725.1$ (SUS) and $\mathbf{R} \$ 76,133,662.8$ (PHIC). Stages 0, I and II comprised about $4.2 \%$ (SUS) and 1.3\% (PHIC) of the total cost; stages III and IV amounted to $95.8 \%$ and 98.7\% of the total cost, respectively.

CONCLUSION: The diagnosis of malignant melanoma in its initial stages reduces treatment costs generating considerable savings of resources for both National Health System and private healthcare insurance companies. Keywords: Melanoma; Melanoma/cost; Melanoma/therapy; Skin neoplasms; Skin neoplasms/cost; Ultraviolet rays
\end{abstract}

Recebido em 12.09.2008.

Aprovado pelo Conselho Consultivo e aceito para publicação em 12.09.08

* Trabalho realizado na Fundação SobecCan - Hospital do Câncer de Ribeirão Preto - Ribeirão Preto (SP) e Universidade Federal de Itajubá (Unifei) - Itajubá (MG), Brasil Conflito de interesse: Nenhum / Conflict of interest: None Suporte financeiro: Nenhum / Financial funding: None

Mestre. Fundação SobecCan - Hospital do Câncer de Ribeirão Preto (SP), Hospital do Câncer de Franca - Franca (SP), Brasil

Mestre e doutora. Departamento de Matemática e Ciências da Computação do Instituto de Ciências Exatas (DMC/ICE), Universidade Federal de Itajubá (Unifei) - Itajubá (MG), Brasil.

Mestre e doutor. Departamento de Ciências Exatas (DCE) - Universidade Federal de Alfenas - Alfenas (MG), Brasil.

Mestre e doutor. Instituto de Recursos Naturais (IRN), Universidade Federal de Itajubá (Unifei) - Itajubá (MG), Brasil.

Graduanda em Administração de Empresas - Universidade Federal de Itajubá (Unifei) - Itajubá (MG), Brasil.

(C)2009 by Anais Brasileiros de Dermatologia

An Bras Dermatol. 2009;84(3):237-43. 


\section{INTRODUÇÃO}

O melanoma origina-se dos melanócitos, células da pele produtoras de um pigmento denominado melanina, que lhe proporciona proteção contra os danos causados pela radiação ultravioleta (UV). O desenvolvimento do melanoma é consequência da perda dos mecanismos genéticos de controle celular causados principalmente pelas radiações UVA e UVB. Entretanto, o melanoma do tipo acral lentiginoso, que compreende aproximadamente $10 \%$ dos casos de melanoma e surge sobretudo nas palmas das mãos, solas dos pés, região subungueal e digitais, parece apresentar um modelo de crescimento distinto dos demais tipos, provavelmente não influenciado pela exposição à radiação UV. ${ }^{1}$

Os três principais tipos de câncer de pele são o carcinoma de células basais (CCB), o carcinoma de células escamosas (CCE), que constituem o grupo denominado câncer de pele não melanoma (CPNM), e o melanoma cutâneo (MC).

O CCB, segundo dados do Instituto Nacional de Câncer (Inca), é responsável por aproximadamente $70 \%$ dos casos de câncer de pele no Brasil. É o tipo mais frequente e menos grave, raramente disseminando para outros órgãos; entretanto, pode destruir os tecidos a sua volta, atingindo cartilagens e ossos. O CCE, o segundo tipo de câncer de pele mais frequente, responsável por aproximadamente $25 \%$ dos casos, possui maior facilidade para disseminar para os gânglios linfáticos e outros órgãos, levando ao surgimento de metástases. Embora as taxas de mortalidade pelos CCB e CCE sejam baixas, esses cânceres podem causar consideráveis deformidades físicas se não tratados. O MC, apesar da menor ocorrência ( $\pm 5 \%$ ), é o mais agressivo, sendo responsável por, aproximadamente, $75 \%$ de todas as mortes causadas por cânceres de pele; esse tipo pode facilmente disseminar para os gânglios linfáticos e órgãos internos.

O diagnóstico precoce é importante para todos os casos, mas particularmente para o MC, pois, nesse caso, o melanoma in situ pode ser curável, mas, iniciada a metástase, torna-se praticamente fatal. Segundo Berwick et al. (1996), ${ }^{2}$ um estudo de casocontrole, baseado em população, mostrou que o autoexame da pele pode reduzir as mortes por câncer de pele, identificando-o precocemente. Também Rocha et al. $(2002)^{3}$ concluem que "o diagnóstico precoce contribui significativamente para a redução da morbimortalidade da doença". No caso do CPNM, o tratamento mais comum é o cirúrgico e, ainda, apresenta o problema de deixar cicatrizes e probabilidade de atingir as estruturas vizinhas.

$\mathrm{O}$ número total de casos de câncer de pele, $\mathrm{MC}$ e CPNM, está sofrendo aumento acelerado mundial- mente. ${ }^{4,5}$ No Brasil, segundo dados do Inca, o mais frequente entre todos os cânceres no país é o de pele, correspondendo a quase $30 \%$ dos casos estimados em 2006. ${ }^{6}$ Ainda de acordo com essa fonte, esses dados podem estar subestimados pelo fato de muitas lesões suspeitas serem retiradas sem diagnóstico ou em consultórios particulares.

Preocupante também é a constatação de que a taxa de crescimento dos cânceres de pele evoluiu cerca de $113 \%$ entre 2001 e 2006 , só ficando atrás da que corresponde ao câncer de próstata. É importante ressaltar que os dados de 2004 não estiveram disponíveis para este levantamento, estando, portanto, fora desta análise. Seguindo essa tendência, serão cerca de 280 casos por 100 mil habitantes em 2010, e aproximadamente 595,92 casos por $100 \mathrm{mil}$ habitantes em 2015.7 Além disso, embora tenha baixa incidência ( $4 \%$ dos casos de câncer de pele), o MC está associado a significativa morbidade, devido a sua alta possibilidade de metástase. ${ }^{6}$ Essas razões justificam as campanhas e os investimentos para prevenção ao câncer de pele que devem ser empreendidos tanto por médicos quanto pelos serviços públicos.

Os fatores de risco para o desenvolvimento do câncer de pele tanto podem ser genéticos quanto ambientais. A causa ambiental mais significante é a exposição excessiva ao sol, particularmente nos primeiros 20 anos de vida. ${ }^{8}$ Pessoas com determinadas características físicas, em especial de pele e olhos claros, apresentam maior risco de desenvolver câncer de pele. ${ }^{9}$ No Brasil, a maior parte desses casos ocorre nas regiões Sul e Sudeste do país, cuja população é predominantemente branca e, portanto, mais susceptível à influência dos altos níveis de R-UV registrados. ${ }^{10}$

$\mathrm{Na}$ população negra, em muitos casos, as lesões iniciais passam despercebidas na pele escura, e o diagnóstico é feito em estádios mais avançados. Além disso, essa população também está mais sujeita ao melanoma acral lentiginoso, não associado à exposição à radiação UV, sendo sua ocorrência maior na Região Nordeste.

Existe outrossim o senso comum de que o padrão estético e socioeconômico predominante é ter a pele bronzeada. Até a Revolução Industrial, a população mais pobre trabalhava na lavoura e estava mais exposta ao sol, enquanto a de nível econômico mais alto procurava manter a pele clara como sinal de status social. Com o surgimento das fábricas, houve migração dos trabalhadores rurais para o parque industrial e, a partir de então, o padrão se inverteu, passando a ser desejável possuir a pele bronzeada como símbolo de condição econômica mais alta. Essa cultura, inserida em um país tropical, que favorece 


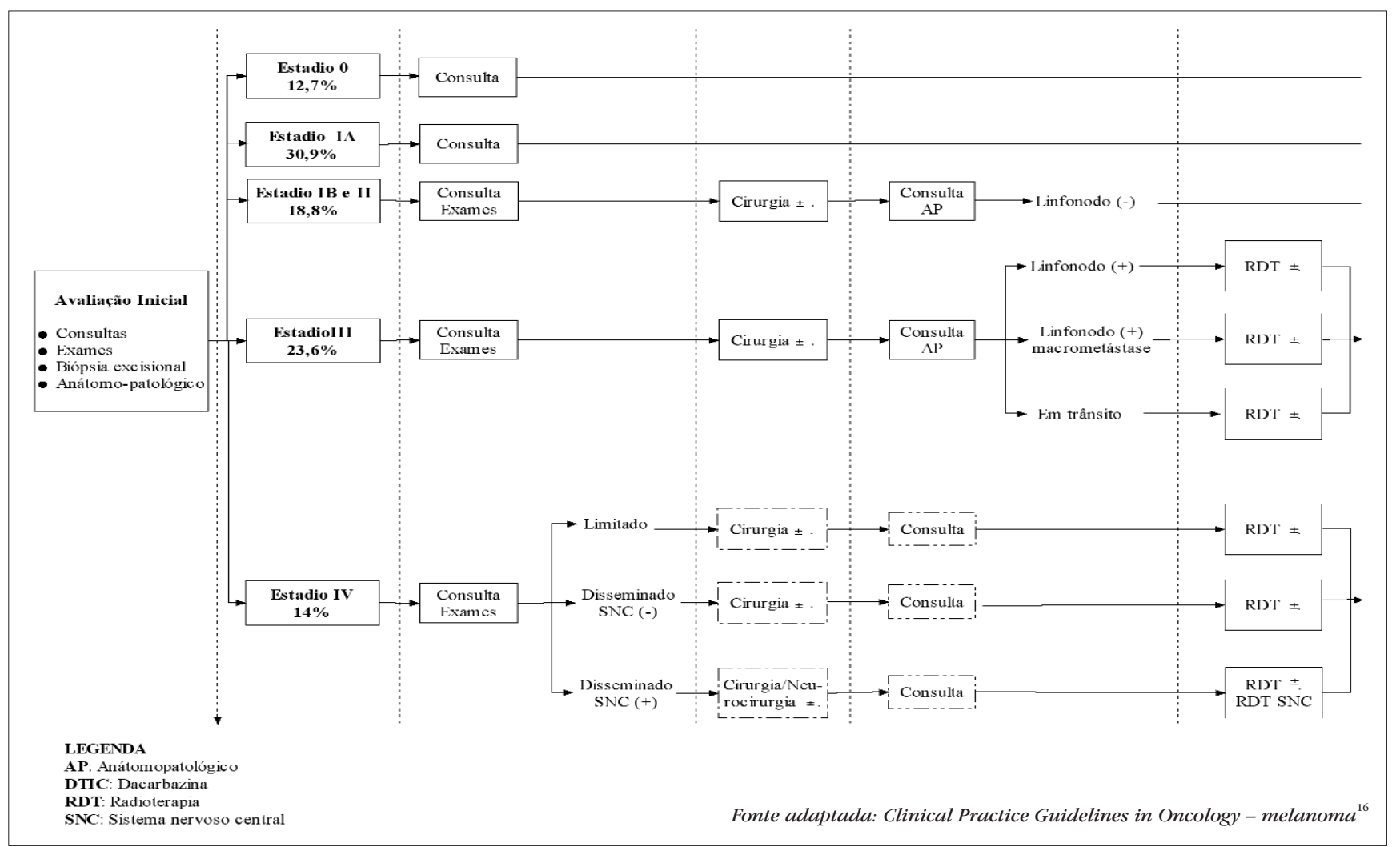

Figura 1: Fluxograma do tratamento do melanoma cutâneo

atividades e esportes sob o sol, induz à excessiva exposição à radiação solar ultravioleta, em especial entre jovens. Pesquisas mostram que, apesar do conhecimento sobre os riscos da exposição excessiva à radiação ultravioleta e sobre as práticas visando à proteção da pele, prevalece entre os jovens o hábito de exposição intencional ao sol. ${ }^{11}$ Segundo os autores, "essa exposição ocorre não somente com o intuito de adquirir um bronzeado, mas também pela despreocupação com a proteção da pele durante atividades cotidianas". Paralelamente, trabalhadores rurais ou do comércio informal comumente observado nas ruas dos grandes centros urbanos também constituem grupo de risco, por desconhecimento dos efeitos do sol ou por dificuldades inerentes a condições socioeconômicas.

Estudos mostram que, nos Estados Unidos, o MC é diagnosticado, em sua maioria, em adultos com idade entre 40 e 50 anos. ${ }^{12}$ No Brasil, o Banco de Dados do Sistema Único de Saúde (Datasus) mostra que o maior número de incidências de MC ocorre acima dos 70 anos, ${ }^{13}$ aproximadamente 0,0021\% dessa população. Entretanto, é possível verificar número significativo de diagnósticos da doença já na faixa etária entre 10 e 29 anos de idade.
Dessa forma, uma vez que crescem tanto a incidência quanto a preocupação com o câncer de pele, é importante que seja avaliado o impacto econômico do exame, diagnóstico, tratamento e acompanhamento do MC. Nesse sentido, o objetivo deste trabalho é fazer um levantamento dos custos relacionados ao tratamento do MC por paciente nos diferentes estádios, sob a perspectiva de dois pagadores: público - via Sistema Único de Saúde (SUS) - e privado - mediante convênios/seguradoras de saúde -, com vistas a avaliar o impacto da doença. Como estudo de caso, serão apresentados os dados referentes ao Estado de São Paulo, no período de janeiro de 2000 a dezembro de 2007.

\section{MÉTODOS}

A pesquisa sobre os custos com o câncer de pele será realizada em algumas etapas, sendo esta a primeira, qual seja a estimativa de custo do tratamento do MC. Um modelo apontando as probabilidades de ocorrência dos diferentes estádios foi criado utilizando os dados do Estado de São Paulo. O passo seguinte será ampliar o estudo para todo o Brasil e incluir os casos de CPNM. Para melhor acurácia do modelo, deverá ser construído um banco de dados geográficos, com informações sobre: histórico regional da doença, indicadores socioeconômicos dos 
pacientes, efetividade de tratamento nos diversos estádios e outras informações que, durante o estudo, se demonstrarem necessárias. Por fim, será possível incluir o cálculo do custo/efetividade ${ }^{*}$ no modelo.

Neste estudo, considerou-se, primeiro, o tratamento de um único paciente, tratado sob a perspectiva de pagadores público (SUS) e privado (convênios) no Brasil. Dessa forma, considerando o sistema público como pagador, as informações financeiras provieram da Tabela de Procedimentos do SIA/SUS e SIH/SUS (ambulatorial e hospitalar, respectivamente), ${ }^{14,15}$ e para o sistema privado foram utilizadas as tabelas de procedimentos AMB92. Os custos unitários foram estimados pelo produto do custo unitário de cada procedimento realizado pela quantidade de cada item utilizado. Depois, foram calculados os valores totais para cada estádio, considerando o número de casos classificados nos diferentes grupos. Os dados são expressos em reais (R\$), valores de 2007 , tanto para o pagamento pelo SUS quanto para convênios, para os quais também é apresentado o valor em $\mathrm{CH}$ (coeficiente de honorários médicos), unidade utilizada pela Associação Médica Brasileira, que quantifica cada procedimento médico para efeito de remuneração. Neste estudo, o valor do $\mathrm{CH}$ foi considerado $\mathrm{R} \$ 0,28$, que corresponde à média dos valores pagos pelos convênios aos procedimentos médicos realizados na Fundação SobecCan - Hospital do Câncer de Ribeirão Preto - SP.

A fim de padronizar a análise dos tratamentos, assumiu-se que todos os novos pacientes com suspeita de câncer de pele se submetem a uma fase de diagnóstico que incorre em custos para: a) consulta ao médico clínico; b) procedimento de biópsia excisional; e c) relatório de patologia. A partir desse procedimento, o modelo apresenta duas possibilidades: câncer de pele do tipo CPNM (não tratado neste estudo) ou câncer de pele do tipo MC. No caso do MC, um fluxograma simplificado com os procedimentos para o diagnóstico e tratamento foi criado a fim de estimar o custo do tratamento e está apresentado na figura 1 . O modelo de procedimento é baseado nas diretrizes do Clinical Practice Guidelines in Oncology, para o melanoma, editado pelo National Comprehensive Cancer Network (NCCN) ${ }^{16}$ que foi adequado aos procedimentos da Fundação SobecCan - - Hospital do Câncer de Ribeirão Preto - SP. O primeiro nível do modelo apresenta a porcentagem de pacientes em vários estádios. Os dados são provenientes da Fundação Oncocentro de São Paulo (Fosp): foram 3.187 diag- nósticos de MC no Estado de São Paulo, entre janeiro de 2000 e dezembro de 2007 , com 2.740 casos já classificados por estadiamento 0 , I, II, III e IV, de acordo com o critério da American Joint Comittee on Cancer (AJCC), e 447 casos não estadiados.

A seleção de tratamento para o MC depende da extensão do câncer, classificada por estádios. Segundo a metodologia definida pela AJCC: estádio Ia: localizado, espessura $\leq 0,75 \mathrm{~mm}$; estádio Ib: localizado, espessura de $0,76 \mathrm{~mm}$ a $1,5 \mathrm{~mm}$; estádio IIa: localizado, espessura de $1,51 \mathrm{~mm}$ a $4 \mathrm{~mm}$; estádio IIb: localizado, espessura > 4mm; estádio III: metástase em trânsito ou apenas envolvimento regional do nódulo; e estádio IV: presença de metástase a distância.

Para o cálculo do custo foram considerados os procedimentos tidos como padrão para o diagnóstico, tratamento e seguimento dos pacientes com MC. Estão incluídos nesses cálculos os custos com consultas médicas, exames laboratoriais, biópsia excisional, exame anatomopatológico, exames de imagem para estadiamento (radiografias, tomografias), procedimentos cirúrgicos complementares e exames pré-cirúrgicos, tratamento medicamentoso do melanoma (interferon, quimioterapia) e a radioterapia paliativa no estádio IV.

Não foram incluídos nesta análise as terapias alternativas, a radioterapia que pode estar indicada em diversas situações (doença residual, lesões inoperáveis, paciente idoso ou debilitado, etc.), diárias hospitalares, medicações diversas não utilizadas diretamente no tratamento do MC nem os custos indiretos como, por exemplo, o tempo despendido pelo paciente com o tratamento ou o tempo improdutivo por afastamento. Além disso, não estão contabilizados, ainda, os custos com as lesões suspeitas, mas que não foram diagnosticadas como MC.

Uma vez que o objetivo do trabalho é estimar o custo direto do tratamento do MC para os diversos estádios, foram utilizados para fins de cálculo os custos produzidos pelo paciente em um ano de tratamento e/ou seguimento, sem ser considerada a ocorrência de óbito durante esse período. Tais particularidades deverão ser detalhadas em etapas posteriores, em cálculos de custo/efetividade.

Novamente, é importante ressaltar que esses valores não refletem necessariamente todos os custos envolvidos, pois englobam aqueles cobrados para prestação de serviços de uma parte do sistema de saúde. Por essa razão, os valores apresentados provavelmente subestimam seu verdadeiro impacto econômico.

\footnotetext{
* Custo/efetividade é assim definido pela Organização Pan-Americana da Saúde: "relação entre o custo e a efetividade, entendendo-se por grau de efetividade o nível de contribuição de um programa ou outra atividade na consecução de metas e objetivos fixados para reduzir as dimensões de um problema ou melhorar uma situação insatisfatória. A análise de custo/efetividade tem por objetivo medir o custo relativo das diversas formas possíveis para a consecução de um objetivo e avaliar se o resultado máximo foi obtido utilizando o mínimo de recursos possível".
} 


\section{RESULTADOS}

Neste estudo foram considerados 3.187 casos de MC diagnosticados no Estado de São Paulo entre janeiro de 2000 e dezembro de 2007. Destes 3.187, 447 não foram estadiados e, por essa razão, não estão incluídos nos cálculos do custo.

O custo direto unitário de tratamento por estádio do MC, pelo SUS e pelo convênio, é apresentado na tabela 1. Chama a atenção, nesses dados, a diferença entre os valores de custos de tratamento dos três primeiros estádios (0, I e II) e os dois últimos. Isso ocorre porque os estádios III e IV requerem tratamentos medicamentosos cujos custos são altos. Todos os estádios apresentam procedimento de investigação e diagnóstico, e é nesse período que o estadiamento do MC é definido. Em seguida, excetuando o estádio 0, uma segunda cirurgia por vezes é necessária para a ampliação das margens cirúrgicas e garantir que todo o tecido afetado tenha sido removido. Os estádios III e IV apresentam o tratamento medicamentoso após essa cirurgia, em geral com interferon, se estádio III, e quimioterapia, se estádio IV.

Na tabela 1 observam-se, também, o número de casos de MC e os custos totais e médios do tratamento por estádio. Novamente, chamam a atenção os altos valores referentes aos estádios III e IV, o que é mais visível no gráfico 1, em que a participação dos gastos com o tratamento em cada estádio com relação ao total é apresentada em detalhes.

A média do custo anual do tratamento por paciente, considerando 2.740 casos, com base nos valores pagos pelo SUS foi de $\mathrm{R} \$ 13.062,40 \pm$ $16.848,60$ e, pelos convênios foi de $\mathrm{R} \$ 26.668,30 \pm$ 42.749,59. Os valores altos dos desvios-padrão, tanto para o sistema de pagamento do SUS quanto para convênios são consequência da grande diferença entre os custos de tratamento dos três primeiros estádios e dos dois últimos. É importante ressaltar os valores totais gastos tanto no sistema público $(\mathrm{R} \$ 33.012 .725,10)$ quanto no privado

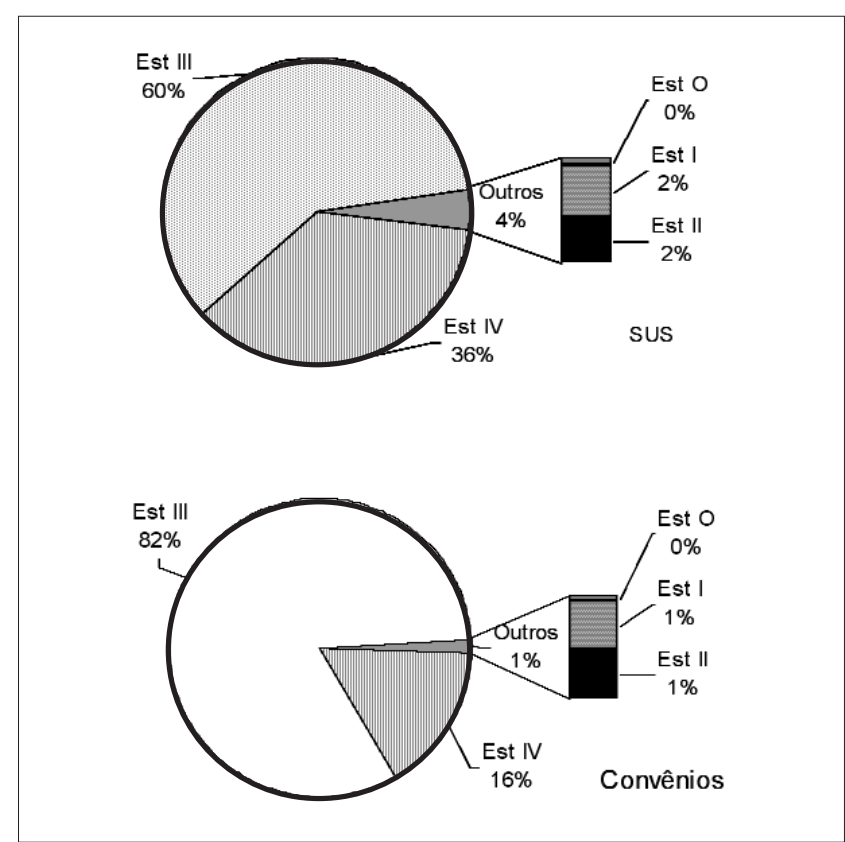

Gráfico 1: Proporção dos custos por estádios nos sistemas público e privado, no Estado de São Paulo, no período de janeiro de 2000 a dezembro de 2007

$(\mathrm{R} \$ 76.133 .662,80)$, que se traduzem em custo médio anual de $\mathrm{R} \$ 4.126 .590,60$ e $\mathrm{R} \$ 9.516 .707,90$, respectivamente.

Esse aspecto fica ainda mais evidente na tabela 2, em que são apresentados os números de casos de câncer de pele estádios 0 e I que podem ser tratados com o valor do tratamento de um único caso de estádio III ou estádio IV. Reitera-se, assim, a importância da prevenção do câncer de pele.

O gráfico 2 apresenta a proporção dos custos das fases de tratamento em cada estádio e nos dois sistemas de pagamento: (a) público e (b) privado. Pode-se notar que, à medida que aumenta o grau de seriedade do MC, os custos aumentam com o tipo de tratamento: o estádio 0 apresenta o maior custo com o diagnóstico; nos estádios I e II , o maior custo refere-se ao tratamen-

TABela 1: Custo unitário e total do tratamento do MC, por estádio, por ano, no Estado de São Paulo, entre janeiro de 2000 e dezembro de 2007

\begin{tabular}{|c|c|c|c|c|c|}
\hline \multirow[t]{2}{*}{ Classificação estádio } & \multirow[t]{2}{*}{$\mathbf{N}$} & \multicolumn{2}{|c|}{$\begin{array}{c}\text { Custo unitário por ano } \\
(\mathrm{em} R \mathbf{R})\end{array}$} & \multicolumn{2}{|c|}{$\begin{array}{l}\text { Custo total por ano } \\
(\mathrm{em} \mathrm{R} \$)\end{array}$} \\
\hline & & SUS & Convênio & SUS & Convênio \\
\hline Estádio 0 & 351 & 382,84 & 176,40 & $134.376,8$ & $61.916,4$ \\
\hline Estádio I & 847 & 767,06 & 554,96 & $649.699,6$ & $470.051,1$ \\
\hline Estádio II & 534 & $1.138,13$ & 903,00 & $607.761,4$ & $482.202,0$ \\
\hline Estádio III & 636 & $30.969,67$ & $98.956,15$ & $19.696 .712,2$ & 62.936.111,4 \\
\hline Estádio IV & 372 & $32.054,23$ & $32.751,03$ & $11.924 .174,8$ & $12.183 .381,9$ \\
\hline Total & 2,740 & & & $33.012 .725,1$ & $76.133 .662,8$ \\
\hline
\end{tabular}


Tabela 2: Número de casos de estádio 0 e I que podem ser tratados pelos valores do custo de tratamento dos estádios III e IV

\begin{tabular}{llll}
\hline & & Estádio 0 & Estádio I \\
\hline Estádio III & SUS & 81 & 40 \\
& Convênio & 561 & 178 \\
\multirow{2}{*}{ Estádio IV } & SUS & 84 & 42 \\
& Convênio & 186 & 59 \\
\hline
\end{tabular}

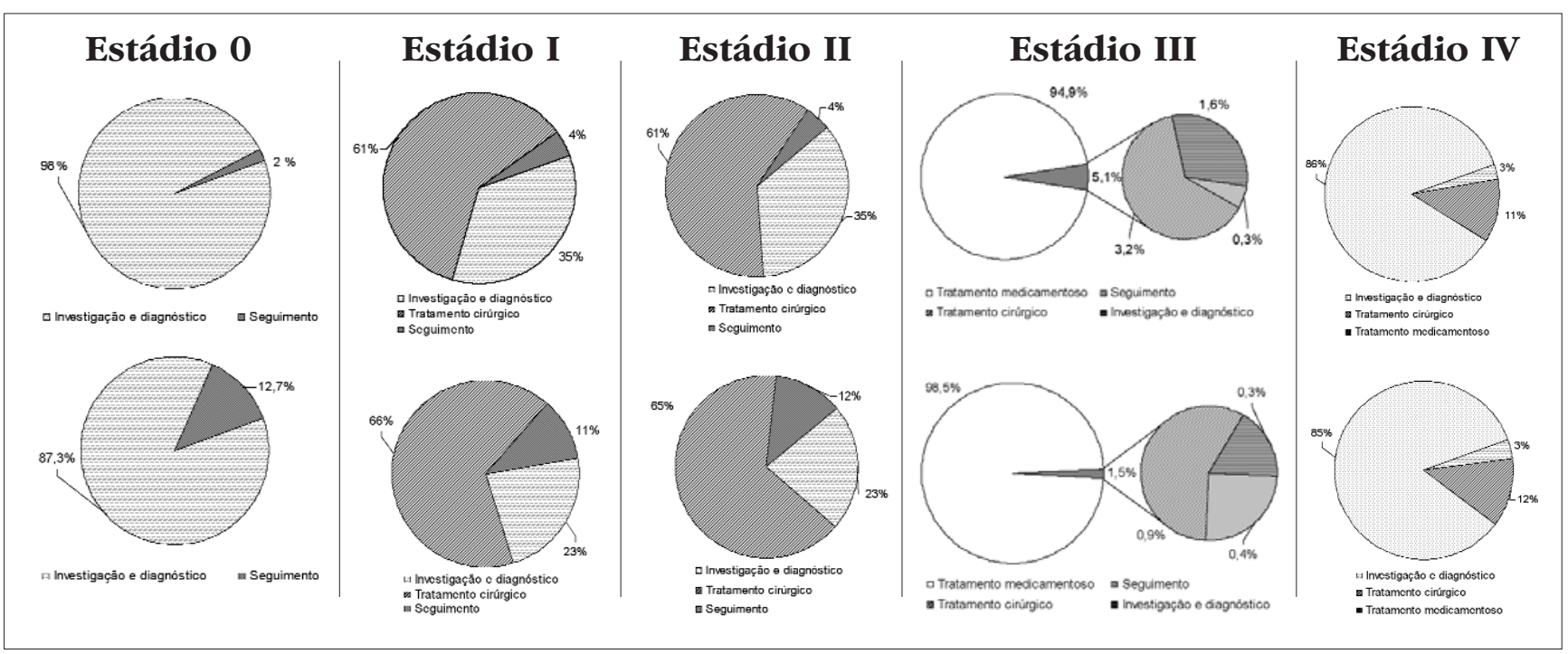

GRáfico 2: Proporção dos custos de tratamento em cada estádio nos sistemas: (a) público e (b) privado (valores de 2007)

to cirúrgico; e nos estádios III e IV predominam os custos com os tratamentos medicamentosos.

\section{DISCUSSÃO}

Grande parte dos custos com o MC, mais de 95\%, é decorrente de tratamentos de estádios mais avançados (III e IV), que demandam medicamentos caros. Nesses casos, o custo do tratamento de um paciente pode cobrir o de vários tratamentos dos estádios iniciais. Demonstra-se, portanto, que a prevenção é importante tanto para salvar vidas quanto por questões financeiras.

Cabe ressaltar que o diagnóstico do melanoma cutâneo em seus estádios iniciais $(0$, I e II) tem também esses efeitos, gerando considerável economia de recursos tanto para o sistema público de saúde (SUS), quanto para o sistema privado (convênios). Esse recurso adicional poderia ser aplicado em programas de prevenção e diagnóstico precoce do câncer de pele ou, ainda, investido em pesquisas de novas técnicas e medicamentos para o tratamento desses pacientes.

\section{CONCLUSÃO}

É senso comum o fato de o câncer de pele gerar gastos diretos e indiretos para governos, planos de saúde e para número significativo de pacientes. Neste estudo, os custos do tratamento do melanoma cutâneo, considerando os procedimentos indicados pela literatura científica e prática da Fundação SobecCan - Hospital do Câncer de Ribeirão Preto - SP, se mostraram elevados, em especial para os tratamentos dos estádios III e IV.

É importante apontar, como ressaltado por Tsao et al.,12 que a análise completa do custo de tratamento de um paciente com câncer de pele "depende de probabilidades definidas pelo histórico natural da doença, o desenvolvimento do exame de pele, a efetividade do tratamento em vários estádios, os riscos de complicações, os riscos competitivos de mortalidade e as perdas de receitas advindas da morte ou da morbidade do tratamento". Para estabelecer um modelo completo, seria necessário banco de dados completo com diversas variáveis que não estão disponíveis no momento. Construir esse banco de dados e desenvolver um modelo mais fidedigno com a realidade serão os próximos passos desta pesquisa. 


\section{REFERÊNCIAS}

1. Liu Z-J, Herlyn M. Molecular biology of cutaneous melanoma. In: DeVita Jr VT, Lawrence TS, Rosenberg SA. Cancer: principle \& practice of oncology. 7 th ed. Philadelphia: Lippincott Williams \& Wilkins, 2005. p. 1746-53.

2. Berwick M, Begg CB, Fine JA, Roush GC, Barnhill RL. Screening for cutaneous melanoma by skin self-examination. J Natl Cancer Inst. 1996;88:17-23.

3. Rocha FP, Menezes AMB, Almeida Junior HL, et al. Especificidade e sensibilidade de rastreamento para lesões cutâneas pré-malignas e malignas. Rev Saude Publica. 2002;36:101-106.

4. Balch CM, Buzaid AC, Soong SJ, Atkins MB, Cascinelli N, Coit DG, et al. Final version of the American Joint Committee on cancer staging system for cutaneous melanoma. J Clin Oncol. 2001;19:3635-48.

5. Diepgen TL, Mahler V. The epidemiology of skin cancer. Br J Dermatol. 2002;146(Suppl 61):S1-6.

6. Brasil, Ministério da Saúde. Secretaria de Atenção à Saúde. Instituto Nacional do Câncer. Coordenação de Prevenção e Vigilância: estimativa 2006: incidência de câncer no Brasil. 2005;1-98.

7. Inca.gov.br [homepage]. Estimativas da incidência e mortalidade por câncer. [acesso 4 Abril 2007]. Disponível em: http://www.inca.org.br.

8. Chinni DA, Schwartz JL, Keilman LJ, et al. Early melanoma detection: what is the role of the advanced practice nurse? The Internet Journal of Advanced Nursing Practice. 2003;5.

9. Barnes L. Sun education in Ireland. Clin Dermatol. 1998; 16:517-8.

10. Corrêa MP, Dubuisson P, Plana-Fattori A. An overview of the ultraviolet index and the skin cancer cases in Brazil. Photochem Photobiol. 2003;78:49-54.
11. Souza SRP, Fischer FM, Souza JMP. Bronzeamento e risco de melanoma cutâneo: revisão da literatura. Rev Saúde Pública. 2004;38:588-98.

12. Tsao H, Rogers GS, Sober AJ. An estimate of the annual direct cost of treating cutaneous melanoma. J Am Acad Dermatol. 1998;38:669-80.

13. W3. datasus.gov.br/datasus/datasus.php [homepage]. Informação de Saúde: Morbidade hospitalar do SUS por local de internação - Brasil. [cited 4 Abril 2007]. Disponível em: http:// tabnet. datasus. gov.br/cgi/tabcgi. exe?sih/cnv/miuf.def

14. W3. datasus.gov.br/datasus/datasus.php [homepage]. Tabela de procedimentos SIH/SUS, Janeiro de 2007. [acesso 2007 April 4]. Disponível em: http://W3. datasus.gov.br/siasih/siasih.php

15. W3. datasus.gov.br/datasus/datasus.php [homepage]. Tabela de procedimentos SIA/SUS, janeiro de 2007. [acesso 4 abril 2007]. Disponível em: http://W3. datasus.gov.br/siasih/siasih.php

16. National Comprehensive Cancer Network (NCCN) Clinical Practice Guidelines in Oncology - melanoma. 2006;V.2.2006:1-33.

ENDEREÇO PARA CORRESPONDÊNCIA / MAILING ADDRESS: Reynaldo José Sant'Anna P. Souza Hospital do Câncer de Franca Av. Presidente Vargas, 2953. Jardim Petráglia 14409055 Franca SP Tel./Fax: $55163712-3070$ / $55163712-3065$. E-mail: reynaldojsantanna@gmail.com / reynaldo@solamigo.org.

Como citar este artigo/How to cite this article: Souza RJSP, Mattedi AP, Rezende ML, Corrêa MP, Duarte EM. Estimativa do custo do tratamento de câncer de pele tipo melanoma no Estado de São Paulo - Brasil. An Bras Dermatol. 2009;84(3):237-43. 